Original research article

\title{
Naringenin alleviates high D-glucose-induced injuries through upregulation of microRNA-30d-5p level in human AC16 cardiac cells
}

\author{
Jiamei Jiang ${ }^{\mathrm{a}}$, Guobiao Liang ${ }^{\mathrm{a}}$, Zijun $\mathrm{Wu}^{\mathrm{a}}$, Hailiang Mo ${ }^{\mathrm{a}}$, Qiong You ${ }^{\mathrm{a}}$, Zhiqiang Wang ${ }^{\mathrm{b}}$, \\ Keng $\mathrm{Wu}^{\mathrm{a}, *}$, Runmin $\mathrm{Guo}^{\mathrm{a}, *}$ \\ a Guangdong Medical University, Affiliated Hospital, Department of Cardiology, Zhanjiang, China \\ ${ }^{\mathrm{b}}$ Guangdong Medical University, Affiliated Hospital, Clinical Research Center, Zhanjiang, China
}

\section{A R T I C L E I N F O}

\section{Article history:}

Received 16 October 2017

Received in revised form 24 January 2018

Accepted 21 February 2018

Available online 2 March 2018

\section{Keywords:}

Diabetes mellitus

Diabetic cardiomyopathy

Naringenin

Variability

Apoptosis

\begin{abstract}
A B S T R A C T
As a common complication of diabetes mellitus (DM), diabetic cardiomyopathy (DCM) is considered to be one of the major causes of mortality and morbidity. The therapeutic effects of naringenin have been verified in the treatment of various human diseases. However, the application of naringenin in the treatment of DCM still has not been reported. In this study, human AC16 cardiac cells were treated with normal D-glucose and high D-glucose (HG). After transfection with miR-30d-5p inhibitor, Cell Counting Kit-8 (CCK-8) method was used to measure cell viability. Hoechst 33258 staining was performed to observe the morphological changes of nucleus. Enzyme-linked immunosorbent assay (ELISA) was performed to determine the activity of caspase-3. Cell apoptosis was detected by Annexin V-FITC/ propidium iodide (PI) staining. Levels of light chain 3 (LC3) including LC3-I and LC3-II as well as nucleoporin p62 (P62) were detected by Western blot. We found that Naringenin treatment increased the reduced cell variability caused by HG treatment. Naringenin also increased expression level of miR-30d5p in human AC16 cardiac cells after HG treatment. Treatment with miR-30d-5p inhibitor reduced the effect of miR-30d-5p in increasing cell variability and reducing cell apoptosis. Naringenin treatment reduced the increased levels of LC-I, LC-II and P62, but miR-30d-5p inhibitor reduced those changes. Therefore we concluded that naringenin could alleviate HG-induced injuries through the upregulation of microRNA-30d-5p level in human AC16 cardiac cells.
\end{abstract}

(c) 2018 Faculty of Health and Social Sciences, University of South Bohemia in Ceske Budejovice. Published by Elsevier Sp. z o.o. All rights reserved.

\section{Introduction}

As a common complication of diabetes mellitus, diabetic cardiomyopathy (DCM) affects about $12 \%$ of patients with diabetes mellitus (Lorenzo-Almorós et al., 2017). DCM is also a leading cause of heart failure and death (Boudina and Abel, 2010). With the changes of people's life style, the incidence of DCM is expected to be significantly increased in the near future, which in turn will increase the incidence of DCM (Bugger and Abel, 2014). Although various treatment strategies have been developed to treat DCM, treatment efficacy is usually unsatisfactory due to the complex pathogenesis (Huynh et al., 2014; Miki et al., 2013). Therefore, the identification of novel treatments with higher efficiency is always needed. As a type of flavonoid extracted from grapefruit and a

\footnotetext{
* Author for correspondence: Guangdong Medical University, Affiliated Hospital, Department of Cardiology, 57 Renmin Avenue, Zhanjiang, Guangdong 524001,
} China. variety of other fruits and herbs, naringenin is proven to be with therapeutic effects in the treatment of various human diseases including various complications of DM (Burke et al., 2015, 2017; Kapoor and Kakkar, 2014). However, the application of naringenin in the treatment of DCM still hasn't been reported.

MicroRNA, or miRNA, is a group of endogenous singlestranded non-coding RNA with a length ranged from 17 to 25 nucleotides. MiRNAs mainly perform their biological functions by targeting the $3^{\prime}$ untranslated region of the target gene to degrade the target gene or inhibit the translation of mRNA (Chen et al., 2012; Reddy, 2015). With the abilities of translational regulation, various miRNAs have been proved to be involved in different biological processes including cell proliferation, migration, apoptosis, organ formation or even tumorigenesis (Shenoy and Blelloch, 2014). As a miRNA, microRNA-30d is proved to be able to interact with foxo3a to improve the conditions of DCM ( $\mathrm{Li}$ et al., 2014) indicating that microRNA-30d may be a new target for the treatment of DCM. 
In this study, DCM model was established by treating human AC16 cardiac cells with high D-glucose. The effects of naringenin treatment on cell variability and apoptosis were detected. The interaction between naringenin and microRNA-30d-5p was also investigated.

\section{Materials and methods}

\section{Cell culture and treatment}

The human AC16 cardiac cell line were obtained from American Type Culture Collection (ATCC, Rockville, MD, USA), cultured in Dulbecco's modified Eagle's medium (Gibco, USA) supplemented containing $100 \mathrm{u} / \mathrm{ml}$ penicillin and streptomycin (Sigma-Aldrich, France) and $10 \%$ fetal bovine serum (Gibco, USA). The cells were grown at $37{ }^{\circ} \mathrm{C}$ in an incubator with $5 \% \mathrm{CO}_{2}$ humidified atmosphere until $60-70 \%$ subconfluent, and then were treated with normal Dglucose $(4.5 \mathrm{mmol} / \mathrm{l})$ or $\mathrm{HG}(33 \mathrm{mmol} / \mathrm{l})$ for different time points $(4,12,24,48$ and $72 \mathrm{~h})$. In order to investigate the effects of cardioprotection of naringenin on HG-induced injury, AC16 cardiac cells were pretreated with naringenin $(10,20$ or $40 \mu \mathrm{m})$ for $2 \mathrm{~h}$ and then co-treatment with $\mathrm{HG}(33 \mathrm{~mm}$ ) for $72 \mathrm{~h}$. In order to determine the role of miR-30d-5p in the cardioprotection of naringenin under HG condition, AC16 cardiac cells were transfected with miR-30d$5 \mathrm{p}$ inhibitors followed by co-treatment with naringenin $(40 \mu \mathrm{m})$ and $\mathrm{HG}$ (33 $\mathrm{mm})$ for $72 \mathrm{~h}$.

\section{Cell viability assay}

Human AC16 cardiac cells (under the logarithmic growth phase) were seeded into 96-well plates with density of $1 \times 10^{4}$ cells/well overnight. After growing to about 70\% confluence, AC16 cardiac cells were treated as described above for $72 \mathrm{~h}$ and the viability of AC16 cells was accessed with CCK- 8 kit according to the manufacture's instruments. After incubated with $10 \mu \mathrm{l}$ of CCK-8 regent for additional $3 \mathrm{~h}$ and the absorbance at $570 \mathrm{~nm}$ was measured with a plate reader (Thermo Fisher Scientific Inc). Each experiment was carried out three times, independently. The cell viability was calculated as the percentage of viable cells in comparison with the control group.

\section{Hoechst 33258 staining}

The morphological changes of nucleus were observed by Hoechst 33258 staining according to the manufacture's instruments. In brief, human AC16 cardiac cells in logarithmic growth phase were seeded into density of $1 \times 10^{5}$ cells/well in 24-well plates. After the treatment which is described above, AC16 cells were washed with $4{ }^{\circ} \mathrm{C}$ PBS for three times, fixed with $4 \%$ paraformaldehyde for $10 \mathrm{~min}$ at $4{ }^{\circ} \mathrm{C}$, and then stained with Hoechst $33258(5 \mu \mathrm{g} / \mathrm{ml})$ for $10 \mathrm{~min}$ at room temperature in dark. The morphology of apoptotic cells was observed by fluorescence microscopy (Bx50-FLA, Olympus, Tokyo, Japan). Apoptotic cells present the phenomenon of pyknosis and are cleavaged with strong blue fluorescence, while normal cells were complete and showed uniform blue fluorescence.

\section{Measurement of caspase-3 activity}

The caspase- 3 activity was determined by caspase- 3 enzyme-linked immunosorbent assay (ELISA) Kits according to the manufacture's instruments. Briefly, human AC16 cardiac cells were seeded into 6 -well plates with the density of $1 \times 10^{6}$ cells/well and were treated with experiment reagent that described above. After lysed by ultrasound (ultrasonic time: $5 \mathrm{~s}$, gap time: $10 \mathrm{~s}$, and circle: 20 times), the cells lysates
$(20 \mu \mathrm{l})$ were co-incubated with a reaction volume $(100 \mu \mathrm{l})$ containing a p-nitroaniline (pNA)-conjugated substrate for caspase-3 (Ac-DEVD-pNA) for $2 \mathrm{~h}$ at $37^{\circ} \mathrm{C}$. The released pNA concentrations were calculated based on the values at $405 \mathrm{~nm}$ and the calibration curve of the defined pNA solutions. The absorbance was measured at $490 \mathrm{~nm}$ on an ELISA plate (Molecular Devices, USA). The Caspase-3 activity in the control group was set as 1 . Each independent experiment was repeated for three times.

Analyses of cell apoptosis by Annexin V-FITC/propidium iodide (PI) staining

The cellular apoptosis level in AC16 cardiac cells were analysed via AnnexinV/PI double staining kit according to the manufacturer's directions. After the treatments, AC16 cells were incubated in binding buffer containing Annexin V-FITC ( $5 \mu \mathrm{l})$ and PI (10 $\mu \mathrm{l})$ at the room temperature for $10 \mathrm{~min}$. The samples were analysed using a flow cytometer (BD FACSAria, Becton-Dickinson, San Jose, CA). The apoptotic ratio was expressed as the ratio between the number of Annexin $\mathrm{V}$-positive cells and the total number of cells counted $\times 100 \%$.

\section{Western blot analysis}

After treatment for $72 \mathrm{~h}$, cells were washed for three times with ice-cold phosphate buffered saline (PBS), lysed in RIPA buffer for $30 \mathrm{~min}$ at $4{ }^{\circ} \mathrm{C}$ (Beyotime), and then centrifugated at $12,000 \times \mathrm{g}$ for $10 \mathrm{~min}$ at $4{ }^{\circ} \mathrm{C}$. The protein concentration was quantified using BCA assay. Equal amounts of protein $(30 \mu \mathrm{g})$ were separated by sodium dodecyl sulphate-polyacrylamide gel electrophoresis (SDS-PAGE) and then transferred to polyvinylidene difluoride (PVDF) membranes. The membranes were blocked with $5 \%$ non-fat milk (Trisbuffered saline (TBST) containing 0.1\% Tween-20 and 5\% milk) at room temperature for $2 \mathrm{~h}$ and then incubated with primary antibodies against LC3, Beclin-1, p62 and Tubulin at 1:2000 dilutions in TBST containing $3 \%$ milk at $4{ }^{\circ} \mathrm{C}$ overnight, respectively. After being washed with TBST for three times, the membranes were incubated with appropriate HRP-conjugated secondary antibodies (Proteintech, USA) at 1:5000 dilutions for $2 \mathrm{~h}$ at the room temperature. Finally, the membranes were processed for an enhanced chemi-luminescence (ECL) kit (Amersham Biosciences, UK). The expression levels of protein were quantified by image v1.48 (NIH, USA). Each of the experiments was repeated for three times, independently.

\section{Statistical analysis}

All data were expressed as the means \pm standard error of the mean (SEM). The difference between two groups was measured by one-way analysis of variance (ANOVA) using SPSS17.0 software (Chicago, IL, USA), and followed by the least significant difference (LSD) test. $P$-value $<0.05$ was considered as significant.

\section{Results}

Naringenin protects AC16 cardiac cells against HG-induced cytotoxicity and apoptosis

Our previous study proved that naringenin has cardioprotection function. In present study, we also found that $\mathrm{HG}(33 \mathrm{~mm}$ ) treatment decreased the viability of AC16 cardiac cells, while pretreatment with naringenin $(40 \mu \mathrm{m})$ blocked this effect (Fig. 1A). Hoechst 33258 staining result revealed that naringenin pretreatment improved the morphological changes of apoptotic cells 
A

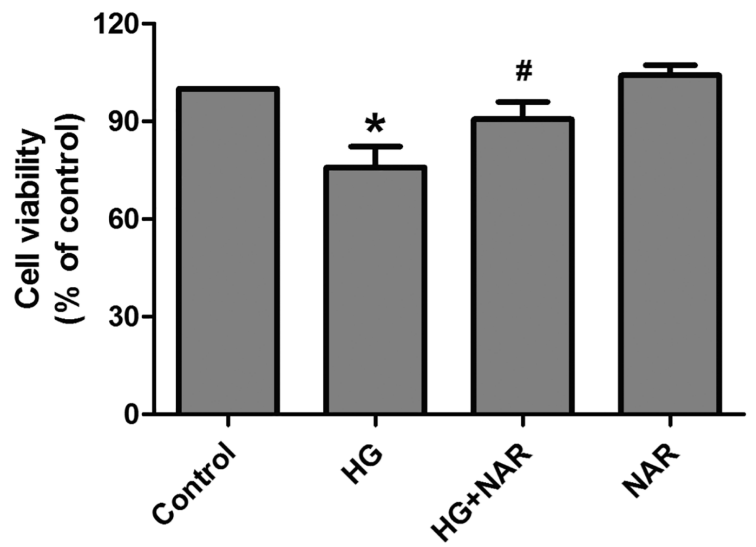

C

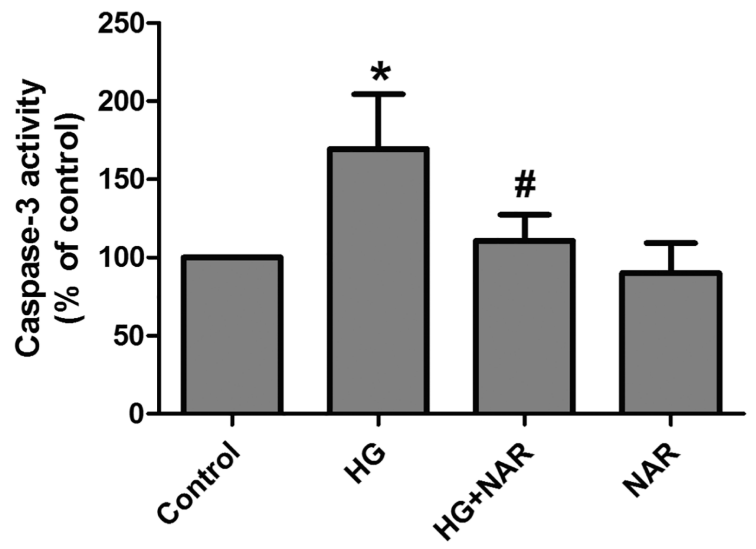

E

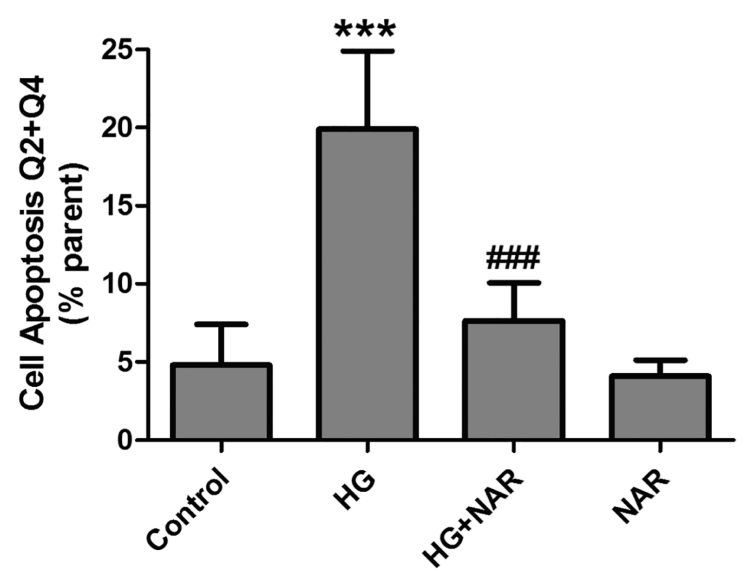

B
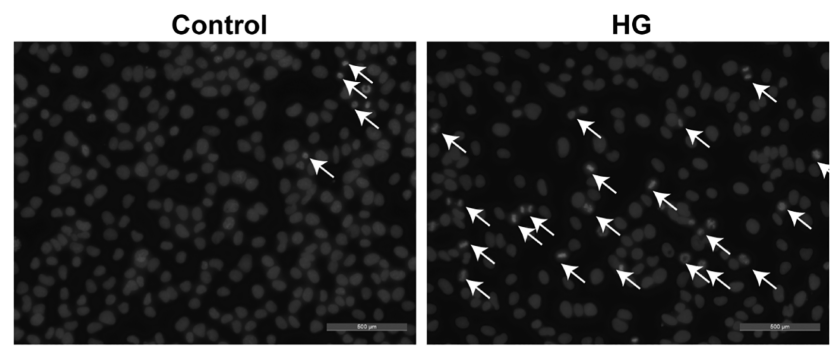

HG+NAR
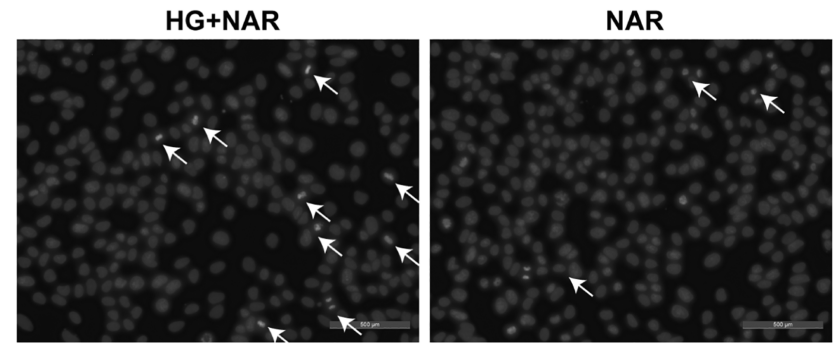

D
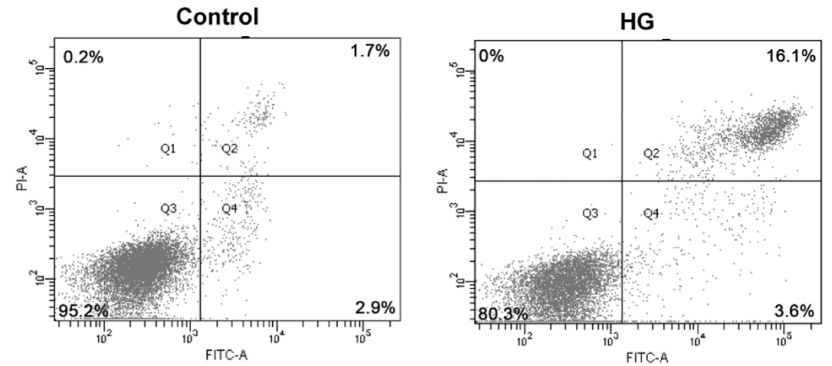

HG+NAR

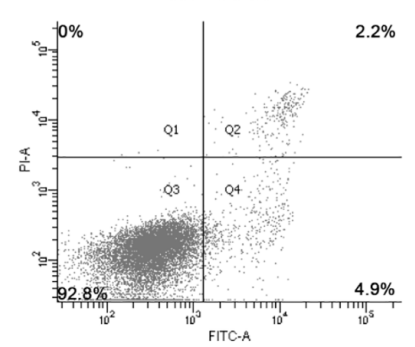

NAR

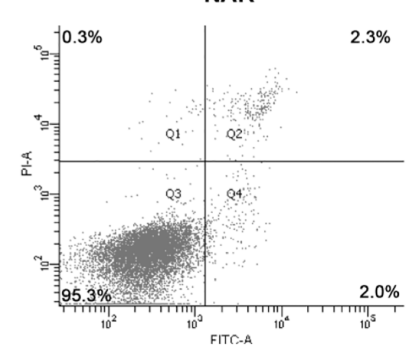

Fig. 1. Cardioprotection of naringenin on $\mathrm{H} / \mathrm{R}$-induced cytotoxicity and apoptosis in human AC16 cardiac cells. AC16 cardiac cells were pre-incubated with naringenin ( 40 $\mu \mathrm{m}$ ) for $2 \mathrm{~h}$ and then exposed to high D-glucose $(33 \mathrm{~mm}$ ) co-treatment for $72 \mathrm{~h}$. Control cells were treated with high glucose (4.5 mm) for $72 \mathrm{~h}$. (A) The cell viability was assayed by CCK-8 assay. (B) The morphological changes of apoptotic cell were observed by Hoechst 33258 staining $(\times 200)$. (C) The caspase-3 activity was measured by the Caspase-3 Assay Kit according to the manufacturer's instructions. Data were expressed as fold of fluorescence intensity in control group. (D) The apoptosis ratio was detected by Annexin V-FITC/PI staining. (E) Statistical analysis from Annexin V-FITC/PI staining results. Data are presented as the mean \pm standard error of the mean $(n=3)$. ${ }^{*} P<0.05$, ${ }^{* * *} P=0.001$ compared with control group; $\# P<0.05$, \#\#\# $=0.001$ compared with HG treatment group. HG: High D-glucose, Naringenin: NAR.

induced by HG treatment in AC16 cardiac cells (Fig.1B). In addition, pretreatment with AC16 cardiac cells with naringenin $(40 \mu \mathrm{m})$ remarkably abolished HG-induced increasing in the activity of caspase-3 (Fig. 1C). Annexin V-FITC/PI staining result (Fig. 1D) also showed that HG increased the apoptosis ratio, while pretreatment with naringenin decreased the apoptosis ratio (Fig. 1D). Naringenin treatment alone did not affect the cells viability and apoptosis. These results suggested that naringenin alleviates cytotoxicity and apoptosis were induced by HG exhibiting cardioprotection under DCM. 
A
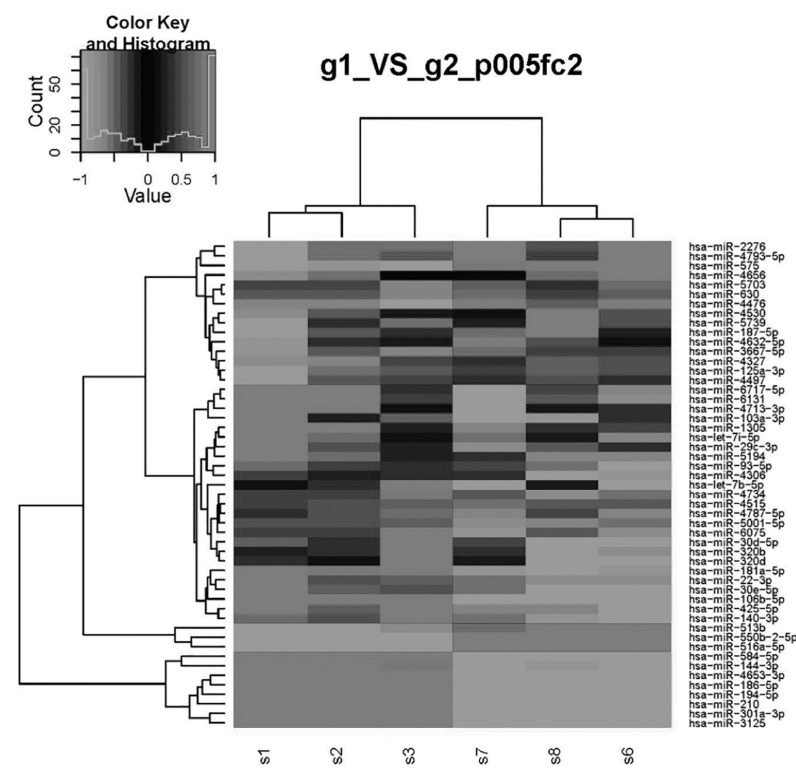

C

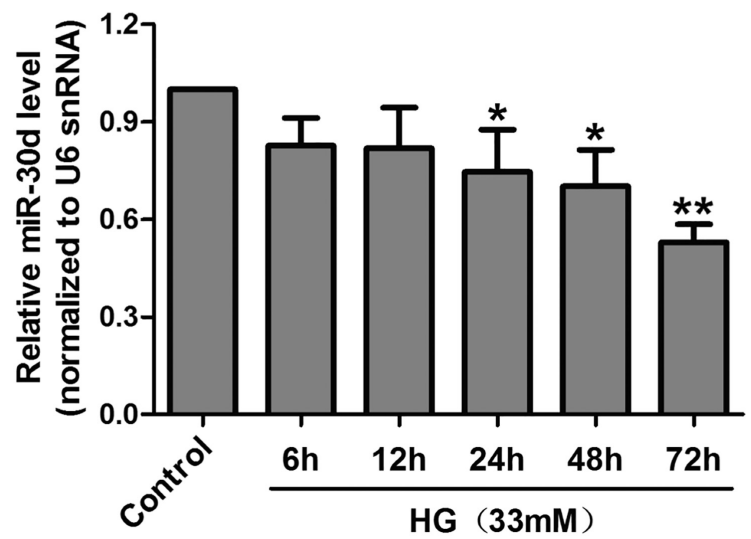

B

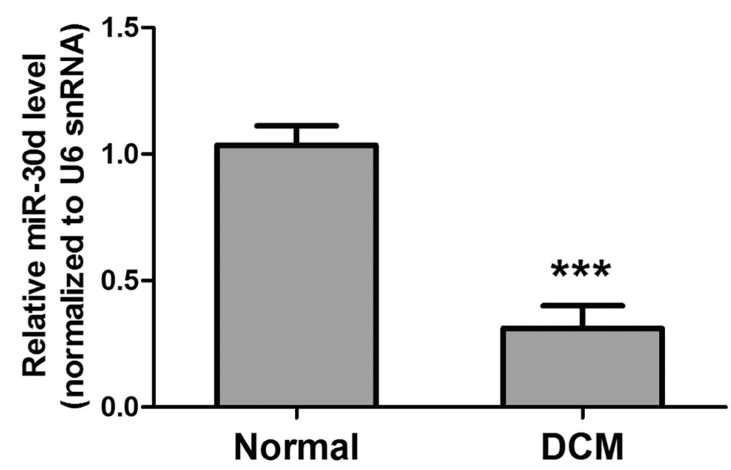

D

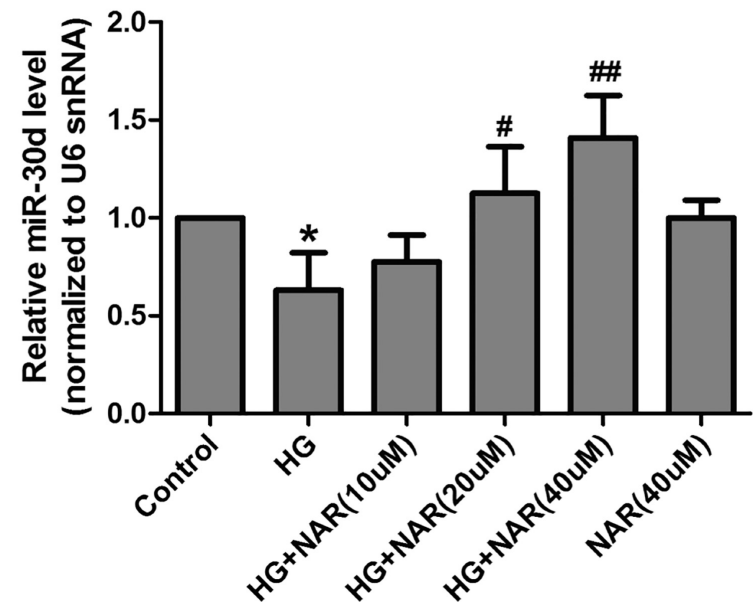

Fig. 2. Effects of naringenin on miR-30d-5p level under HG condition in human AC16 cardiac cells. (A) The levels of microRNAs in the serum of Diabetic cardiomyopathy (DCM) patients $(n=3)$ and normal people $(n=3)$ were detected by hierarchical clustering. (B) The level of miR-30d-5p in the serum of DCM patients $(n=13)$ and normal people $(n=7)$ was measured by real-time quantitative PCR (qRT-PCR). (C) AC16 cardiac cells were treated with HG ( 33 mm) for different time periods ( $6,12,24,48$ and $72 \mathrm{~h}$ ), and the level of miR-30d-5p was assessed by RT-qPCR. (D) AC16 cardiac cells were pre-incubated with naringenin ( $40 \mu \mathrm{m})$ for $2 \mathrm{~h}$ and then exposed to HG (33 mm) co-treatment for $72 \mathrm{~h}$, and the level of miR-30d-5p was assessed by RT-qPCR. Data are expressed as the mean \pm standard error of the mean of three independent experiments. ${ }^{*} P<0.05$ ${ }^{* *} P<0.01,{ }^{* * *} P<0.001$ compared with control group; $\# P<0.05$, \#\#P<0.01 compared with HG treatment group. DCM: Diabetic cardiomyopathy, HG: High D-glucose, Naringenin: NAR.

Naringenin reverses $H G$-induced the down-regulation of miR-30d-5p in human AC16 cardiac cells

Our microRNA microarray screening techniques results showed that the level of miR-30d-5p was remarkably reduced in serum of DCM patients compared to that in the serum of normal person without disease (Fig. 2A). Then, we further used real-time quantitative PCR (qRT-PCR) technique to verify this change and found that the level of miR-30d-5p in serum of patients with DCM was also significantly lower than that in normal subjects (Fig. 2B). In addition, we found that the level of miR-30d-5p in the culture supernatant of AC16 cardiac cells which were treated with HG (33 mm) for 24,48 or $72 \mathrm{~h}$ was also increased compared to that in the culture supernatant of AC16 cardiac control cells that were treated with HG $(4.5 \mathrm{~mm}$ ) (Fig. 2C). Subsequently, in order to investigate whether miR-30d-5p contributes to the cardioprotection of naringenin in DCM, we measured the effect of naringenin on
miR-30d-5p level. QRT-PCR results showed that pretreatment of AC16 cardiac cells with naringenin (20 or $40 \mu \mathrm{m}$ ) significantly reversed HG-induced downregulation of miR-30d-5p level in AC16 cardiac cells (Fig. 2D). Naringenin treatment alone has no effects on miR-30d-5p level in AC16 cardiac cells. These results suggested that miR-30d-5p may be involved in the protection of naringenin on DCM injury or the occurrence of DCM.

miR-30d-5p Inhibitor mitigates the protection of naringenin on HGinduced cytotoxicity in human AC16 cardiac cells

To further confirm the role of miR-30d-5p in the cardioprotection of naringenin against HG-induced injuries, AC16 cardiac cells were transfected with miR-30d-5p inhibitor before cotreatment with naringenin and HG. We found that transfection of miR-30d-5p inhibitor reduced the viability of AC16 cardiac cells comparing with naringenin and HG co-treatment group (Fig. 3). 
A
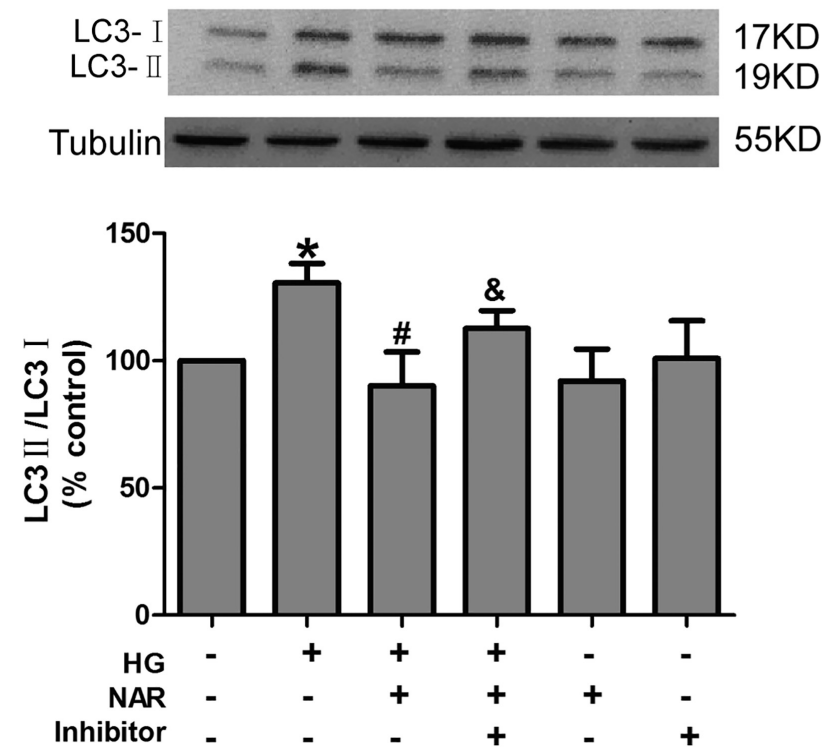

B

P62

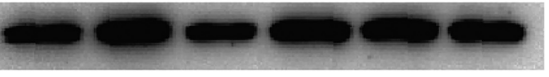

$62 \mathrm{KD}$
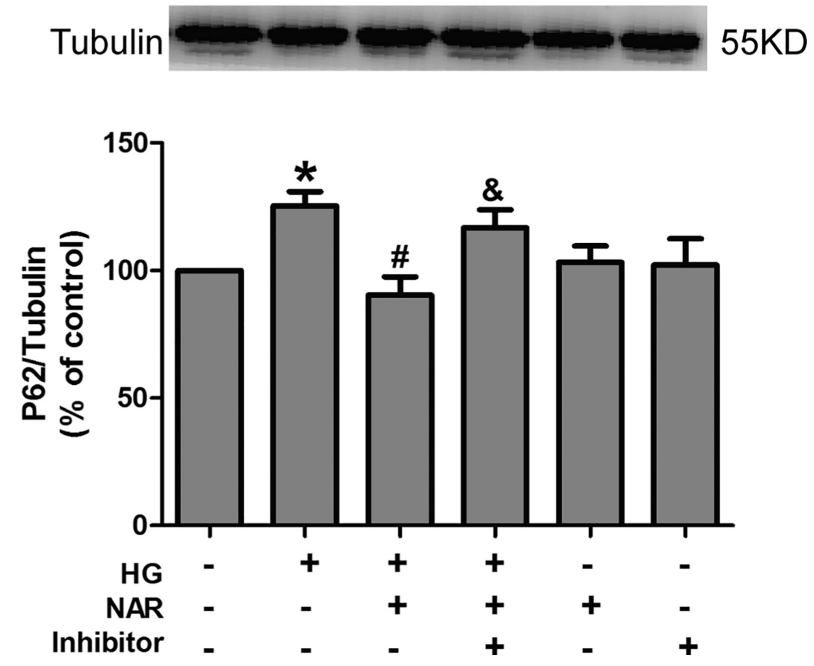

Fig. 3. Effect of miR-30d-5p inhibitor on naringenin-induced the increase in cell viability in HG-treated human AC16 cardiac cells. AC16 cardiac cells were transfected with miR-30d-5p inhibitor or miRNA inhibitor negative control (mimics-NC) before exposure to naringenin (40 $\mu \mathrm{m})$ and $\mathrm{HG}$ ( $33 \mathrm{~mm}$ ) co-treatment for $72 \mathrm{~h}$. The cell viability was assayed by CCK-8 assay. Data are presented as the mean \pm standard error of the mean $(n=3) .{ }^{*} P<0.05$ compared with control group; \#P<0.05 compared with HG treatment group. \&P< 0.05 compared with NAR and HG co-treatment group. HG: High D-glucose, Naringenin: NAR, Inhibitor: miR-30d-5p inhibitor.

MiR-30d-5p inhibitor transfection alone had no effects on cell viability. The results suggested that miR-30d-5p is involved in naringenin-induced cytoprotection against HG-induced AC16 cardiac cells injury.

miR-30d-5p inhibitor abolishes the protection of naringenin on HGinduced apoptosis in human AC16 cardiac cells

Finally, we investigated the effect of miR-30d-5p inhibitor on apoptosis. Hoechst 33258 staining results showed that naringenin ameliorated HG induced the morphological changes of apoptotic cells which were also abolished by miR-30d-5p inhibitor transfection (Fig. 4A). In addition, miR-30d-5p inhibitor reversed naringenin-induced downregulation of caspase-3 activity (Fig. 4B) as well as apoptosis ratio (Fig. 3D and F) in HG-treated AC16 cardiac cells. MiR-30d-5p inhibitor transfection also reversed naringenininduced decrease in apoptosis ratio of HG-treated AC16 cardiac cells. MiR-30d-5p inhibitor transfection alone did not affect apoptosis in AC16 cardiac cells. These results suggested that miR-30d-5p mediated the protection of naringenin against HGinduced apoptosis in AC16 cardiac cells.

\section{Discussion}

It has been well established that the development and progression of DCM are closely related to reducing cell variability and increasing cell apoptosis (Tarquini et al., 2011). In this study, compared with control group, cell variability was significantly decreased and cell apoptosis was significantly increased after HG treatment, indicating the successfully established DCM model. Caspase 9 is a major player in inducing cell apoptosis (Kuida et al., 1998). In this study, activity of caspase 9 was significantly increased in AC16 cardiac cells after HG treatment suggesting that HG treatment can induce cell apoptosis by increasing the activity of caspase 9 . Naringenin is a type of flavonoid with obvious therapeutic effects in the treatment of various human diseases (Burke et al., 2015, 2017; Kapoor and Kakkar, 2014). Using rat as a model, Chtourou et al. (2015) found that naringenin could inhibit cell apoptosis and increase cell variability that was induced by cardiac hypercholesterolemia through reducing oxidative stress.

In this study, naringenin significantly improved cell variability and inhibited cell apoptosis of AC16 cardiac cells with HG treatment. Besides that, naringenin also significantly reduced the increased activity of caspase 9, which was caused by HG treatment. Those results suggest that naringenin treatment can promote the conditions of DCM by increasing cell variability and inhibiting cell apoptosis via inhibiting the activity of caspase 9.

As a miRNA, miR-30d-5p has been proven to be involved in the development of various human diseases. In the study of non-small cell lung cancer, Chen et al. (2015), found that microRNA-30d-5p can interact with CCNE2 to inhibit the proliferation and migration of tumour cells.

In another study, Jia et al. (2016) showed that expression level of miR-30d-5p was significantly reduced in patients with myocardial infarction, and the reduced expression level of miR-30d-5p can be used to accurately predict DCM.

In a recent study, expression level of miR-30d-5p was found to be reduced in patients with DCM, and miR-30d-5p might serve as a novel target for the treatment of DCM (Li et al., 2014). In this study, expression level of miR-30d-5p was significantly decreased in AC16 cardiac cells after the treatment with HG $(p<0.05)$. However, naringenin treatment at a dose of 20 or $40 \mu \mathrm{mol}$ significantly increased the expression level of miR-30d-5p ( $p<0.05$ or $p<0.01)$. In addition, naringenin treatment showed no significant effects on the expression of miR-30d-5p in human AC16 cardiac cells without HG treatment, indicating high safety of applying naringenin in the treatment of DCM. Those data suggest that naringenin treatment can improve the conditions of DCM by upregulating the expression level of miR-30d-5p.

Numerous studies have proven that naringenin can perform its functions by interacting with miRNAs (Curti et al., 2017; Shi et al., 2016). A recent study reported that naringenin could regulate the expression of miR-17-3p to perform its enantioselective modulatory roles in endogenous antioxidant defenses (Curti et al., 2017). In another study, naringenin was found to be able to interact with miR-223 to inhibit activation of neutrophils, which was induced by 
A

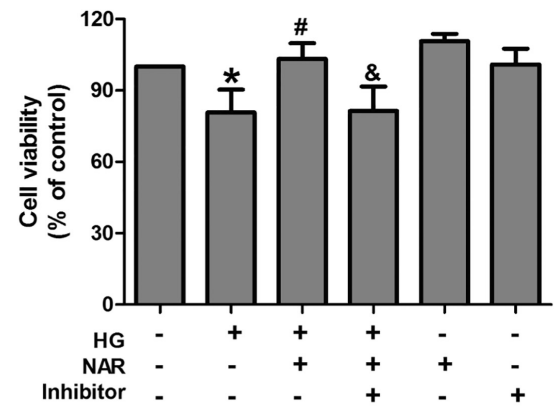

C

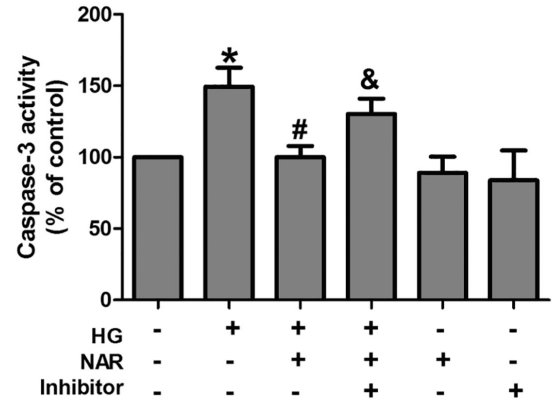

B
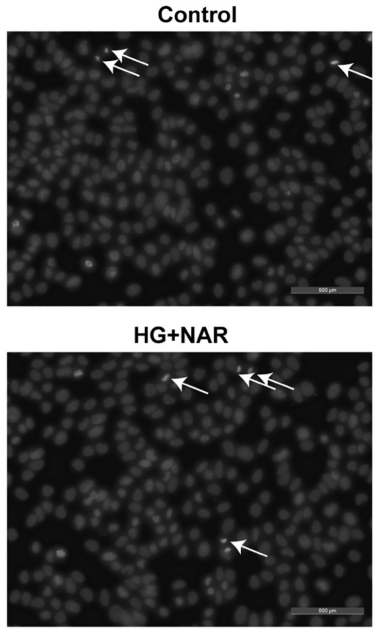

NAR

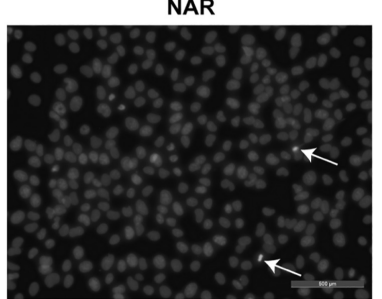

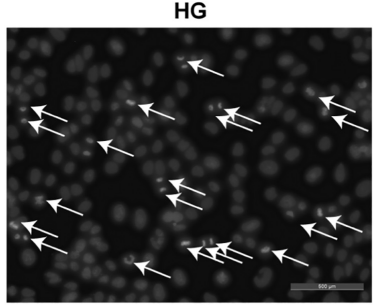

HG+NAR+Inhibitor

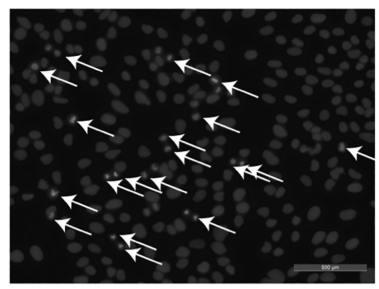

Inhibitor

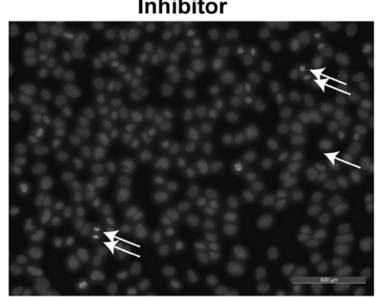

D
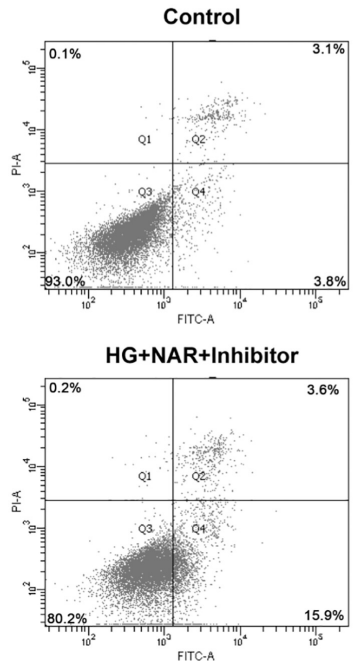

HG
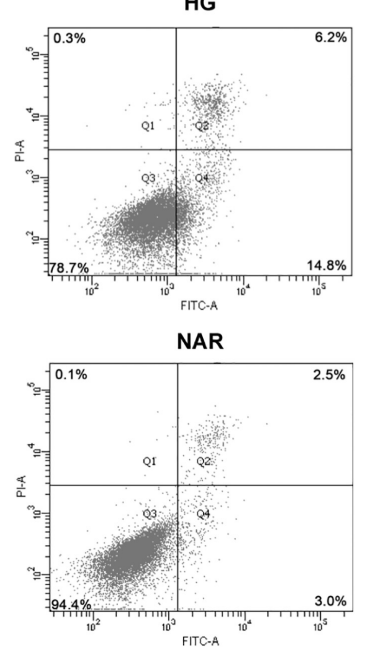

E
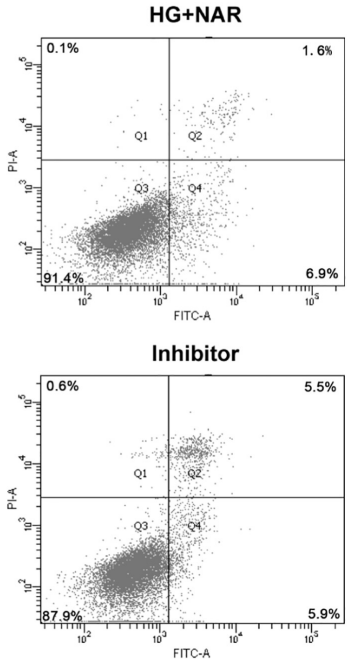

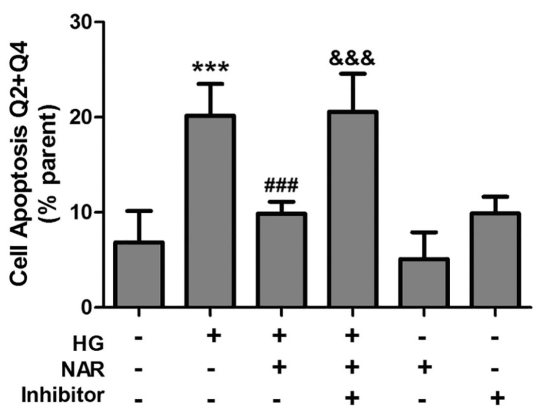

Fig. 4. Effect of miR-30d-5p inhibitor on naringenin-induced the decrease in apoptosis in HG-treated human AC16 cardiac cells. AC16 cardiac cells were transfected with miR30d-5p inhibitor or miRNA inhibitor negative control (mimics-NC) before exposure to naringenin (40 $\mu \mathrm{M})$ and $\mathrm{HG}$ (33 mm) co-treatment for $72 \mathrm{~h}$. (A) The morphological changes of apoptotic cell were observed by Hoechst 33258 staining $(\times 200)$. (B) The caspase-3 activity was measured by the Caspase-3 Assay Kit according to the manufacturer's instructions. Data were expressed as fold of fluorescence intensity in control group. (C) The apoptosis ratio was detected by Annexin V-FITC/PI staining and (D) Statistical analysis from Annexin V-FITC/PI staining results. Data are presented as the mean \pm standard error of the mean $(n=3)$. ${ }^{*} P<0.05$, ${ }^{* * *} P=0.001$ compared with control group; $\# P<0.05$, \#\#\#P=0.001 compared with HG treatment group; $\& P<0.05, \& \& \& P=0.001$ compared with NAR and HG co-treatment group.

spinal cord injury (Shi et al., 2016). However, based on our knowledge, the interactions between naringenin and miR-30d-5p still have not been reported. To further confirm the interactions between naringenin and miR-30d-5p, miR-30d-5p inhibitor was further used to treat AC16 cardiac cells with HG and naringenin treatments. The results showed that miR-30d-5p inhibitor not only reduced increased cell viability of HG-treated human AC16 cardiac cells that were induced by naringenin treatment but also abolished the protection role of naringenin in inhibiting apoptosis caused by HG treatment. Those results suggest that naringenin treatment can improve the conditions of DCM mainly through the upregulation of miR-30d-5p.

In conclusion, this study found that naringenin treatment increased the reduced variability of human AC16 cardiac cells, which was caused by HG treatment. Expression level of miR-30d$5 p$ was reduced in AC16 cardiac cells with HG treatment, while naringenin treatment significantly increased expression level of miR-30d-5p in HG-treated AC16 cardiac cells. Our study also found that miRNA-30d-5p inhibitor reduced the effect of miR-30d-5p in increasing variability and reducing apoptosis of human AC16 
cardiac cells with HG treatment. Based on those data, we may safely conclude that naringenin could alleviate HG-induced injuries through the upregulation of microRNA-30d-5p level in human AC16 cardiac cells. Our study shed new lights into the application of naringenin in the treatment of DCM. However, all experiments in this study were performed in vitro cultured cells. Therefore, future experimental studies with animal models or clinical studies are expected to further confirm the conclusions in this study.

\section{Conflict of interests}

The authors have no conflict of interests to declare.

\section{References}

Boudina, S., Abel, E.D., 2010. Diabetic cardiomyopathy, causes and effects. Rev. Endocr. Metab. Disord. 11 (1), 31-39.

Bugger, H., Abel, E.D., 2014. Molecular mechanisms of diabetic cardiomyopathy. Diabetologia 57 (4), 660-671.

Burke, A.C., Sutherland, B.G., Assini, J.M., Huff, M.W., 2015. Abstract 686: intervention with naringenin enhances weight loss, potentiates improvements in metabolic dysregulation and halts progression of atherosclerosis induced by a high-fat diet in LDLr-/-mice. Arterioscler. Thromb. Vasc. Biol. 35 (1), A686.

Burke, A.C., Telford, D.E., Sutherland, B.G., Edwards, J.Y., Huff, M.W., 2017. Naringenin supplementation to a chow diet reduces plasma lipids and adiposity, and suppresses Rer in Ldlr-/-mice fed a chow diet. Arterioscler. Thromb. Vasc. Biol. 37 (1), A369.

Chen, Y., Zhang, J., Wang, H., Zhao, J., Xu, C., Du, Y., et al., 2012. miRNA-135a promotes breast cancer cell migration and invasion by targeting HOXA10. BMC Cancer 12,111.

Chen, D., Guo, W., Qiu, Z., Wang, Q., Li, Y., Liang, L., et al., 2015. MicroRNA-30d-5p inhibits tumour cell proliferation and motility by directly targeting CCNE2 in non-small cell lung cancer. Cancer Lett. 362 (2), 208-217.
Chtourou, Y., Slima, A.B., Makni, M., Gdoura, R., Fetoui, H., 2015. Naringenin protects cardiac hypercholesterolemia-induced oxidative stress and subsequent necroptosis in rats. Pharmacol. Rep. 67 (6), 1090-1097.

Curti, V., Di Lorenzo, A., Rossi, D., Martino, E., Capelli, E., Collina, S., et al., 2017. Enantioselective modulatory effects of naringenin enantiomers on the expression levels of miR-17-3p involved in endogenous antioxidant defenses. Nutrients 9 (3), 215.

Huynh, K., Bernardo, B.C., McMullen, J.R., Ritchie, R.H., 2014. Diabetic cardiomyopathy: mechanisms and new treatment strategies targeting antioxidant signaling pathways. Pharmacol. Ther. 142 (3), 375-415.

Jia, K., Shi, P., Han, X., Chen, T., Tang, H., Wang, J., 2016. Diagnostic value of miR-30d5p and miR-125b-5p in acute myocardial infarction. Mol. Med. Rep. 14 (1), 184194.

Kapoor, R., Kakkar, P., 2014. Naringenin accords hepatoprotection from streptozotocin induced diabetes in vivo by modulating mitochondrial dysfunction and apoptotic signaling cascade. Toxicol. Rep. 1, 569-581.

Kuida, K., Haydar, T.F., Kuan, C.Y., Gu, Y., Taya, C., Karasuyama, H., et al., 1998. Reduced apoptosis and cytochrome c-mediated caspase activation in mice lacking caspase 9. Cell 94 (3), 325-337.

Li, X., Du, N., Zhang, Q., Li, J., Chen, X., Liu, X., et al., 2014. MicroRNA-30d regulates cardiomyocyte pyroptosis by directly targeting foxo3a in diabetic cardiomyopathy. Cell Death Dis. 5 (10), e1479.

Lorenzo-Almorós, A., Tuñón, J., Orejas, M., Cortés, M., Egido, J., Lorenzo, Ó., 2017. Diagnostic approaches for diabetic cardiomyopathy. Cardiovasc. Diabetol. 16 (1), 28.

Miki, T., Yuda, S., Kouzu, H., Miura, T., 2013. Diabetic cardiomyopathy: pathophysiology and clinical features. Heart Fail. Rev. 18 (2), 149-166.

Reddy, K.B., 2015. MicroRNA (miRNA) in cancer. Cancer Cell Int. 15 (1), 38

Shenoy, A., Blelloch, R.H., 2014. Regulation of microRNA function in somatic stem cell proliferation and differentiation. Nat. Rev. Mol. Cell Biol. 15 (9), 565.

Shi, L.B., Tang, P.F., Zhang, W., Zhao, Y.P., Zhang, L.C., Zhang, H., 2016. Naringenin inhibits spinal cord injury-induced activation of neutrophils through miR-223. Gene 592 (1), 128-133.

Tarquini, R., Lazzeri, C., Pala, L., Rotella, C.M., Gensini, G.F., 2011. The diabetic cardiomyopathy. Acta Diabetol. 48 (3), 173-181. 